\title{
XII.
}

\section{Zur Geschichte des Briefwechsels zwischen Leibniz und Malebranche}

\author{
von
}

Dr. Artur Buchenau-Marburg.

Die Gerhardtsche Ausgabe der "philosophischen Schriften" von Leibniz (Berlin 1875-1890) enthält in ihrem ersten Bande (S. 315 ff.) den Briefwechsel zwischen Leibniz und Malebranche. Der Herausgeber druckt 16 Briefe ab, darunter sind 10 von Leibniz, 6 von Malebranche verfaßt. Dazu kommt noch als „Beilage“ (S. 333 bis 339) der Entwurf eines Leibnizschen Schreibens, das vielleicht für den französischen Philosophen bestimmt war. Abgesehen von dieser Beilage war die Korrespondenz zwischen Leibniz und Malebranche bereits von Victor Cousin zu wiederholten Malen ${ }^{1}$ ) veröffentlicht worden. Cousin hatte sich zu diesem Zwecke Abschriften aus der Königlichen Bibliothek zu Hannover kommen lassen. Gerhardt hat zwar die Originale von neuem verglichen, im übrigen aber nichts geändert. Indessen macht er selbst auf eine Guhrauersche Notiz aufmerksam, deren genauere Verfolgung ihn auf die Spur eines siebzehnten Schreibens geführt haben würde. Diese Notiz ${ }^{2}$ ) lautet: „Von dem Briefwechsel zwischen Leibniz und Malebranche waren trüher nur die beiden Briefe von Malebranche, 13. Dez. 1698, und von Leibniz, 2./12. Okt. 1698 in Feders Specimina com. epist. Leibnitz p. 136-141 bekannt. Herrn Cousin verdanken wir seit

1) Zuerst im „Journal des Savants“ (Juli-Oktober 1844), dann im folgenden Jahre in den "Fragments de Philosophie Cartésienne" (S. 349-428) und schlieSlich in den „Fragments philosophiques" (5. Ausgabe. Paris 1866).

3) Gottfried Wilhelm Freiherr v. Leibniz. Eine Biographie von G. E Guhrauer. Teil I. (Breslau 1846) S. 69 f. der Anmerkungen. 
kurzem die Veröffentlichung oder eigentlich nur dic allgemeinere Verbreitung dieses ganzen Briefwechsels, mit schätzbaren Einleitungen und $\mathrm{Z}$ wischenreden in den Monaten Juli bis Oktober des Journal des Savans von 1844." Daran knüpft Guhrauer die Bemerkung: "Herr Cousin scheint nicht gewußt zu haben, daß die Briefe Leibnitzens an Malebranche, wie an den P. Lelong schon 24 Jahre vorher in Paris, freilich nur in sehr wenig Exemplaren für die Mitglieder der Société des bibliophiles herausgegeben worden sind. Die Notiz gibt Quérard, La France littéraire T. V. p. 119. Paris 1833: , Lettres au P. Malebranche et au P. Lelong. Paris, de l'imprimerie de F. Didot 1820. gr. in -8 de 88 pages. Tirées à 30 exemplaires pour les mélanges de la société des bibliophiles . . . signés Durand de Laucon'. Hier scheinen mehr Briefe von Malebranche zu sein, als die von Cousin herausgegebenen; jedenfalls wird es bei einer künftigen Herausgabe dieser Briefe unerläßlich sein, diese editio princeps zu vergleichen. " Feder hatte in der Tat schon 1805 als erster die zwei oben erwähnten Briefe aus der Korrespondenz zwischen Leibniz und Malebranche mitgeteilt. ${ }^{3}$ ) Er kannte auch die übrigen Briefe, wie aus einer Anmerkung zu S. 138 seiner Ausgabe hervorgeht, glaubte aber wohl, sich auf die „interessantesten" Schreiben beschränken zu dürfen. Trotz der letzten Guhrauerschen Bemerkung haben weder Cousin noch Gerhardt sich um die Auffindung dieser „editio princeps" bemüht. Verfasser dieses fand nun bei einem Ferienaufenthalt in Paris in der „Bibliothèque nationale“. (Inv. réservé 73,265 ) in den „Mélanges publiés par la Société des Bibliophiles français I. Bd. 1820: „Lettres au P. Malebranche et au P. Lelong. Pariș, de l'imprimerie de F. Didot 1820 gr. in : $8^{\circ}$." Diese Ausgabe ist zweifellos identisch mit der oben yon Quérard zitierten. Der Herausgeber, Durand de Lançon - nicht Laucon, wie Guhrauer schreibt -, der selbst Mitglied der "Gesellschaft" ist, schickt folgende Bemerkung voraus:

3) J. G. H. Feder. Lettres choisies/de la|Correspondance|de|Leibnit z. publiées pour la première fois. Hannovre 1805, mit dem lateinischen Nebentitel: Commercii epistolici /Leibnitiani / Typis nondum vulgati|Selecta specimina|edidit|notulisque passim illustravit|Joannes Georgius Henricus Feder (S. 136-141). 
"Des recherches faites à la Bibliothèque du Roi, avec autant d'exactitude que le permet la disette de livres modernes qui se fait sentir dans cet établissement, n'unt pu faire découvrir aucune de ces lettres, soit dans la collection des Euvres de Leibniz, soit dans les diverses parties de sa correspondance, qui ont paru successivement.

Elles sont imprimées sur les lettres autographes qui ont été achetées à la vente des livres du P. Adry, dernier bibliothécaire de l'Oratoire, entre les mains duquel elles sont tombées, lors de la destruction de cette maison.

L'original a été scrupuleusement suivi; on a supprimé seulement quelques passages très-insignifiants, et que ne pourrait regretter l'amateur le plus minutieux de l'histoire littéraire.

Durand de Lançon."

Die Briefe nehmen in Anspruch die Seiten 3-88; das letzte Blatt [87/88] ist doppelt vorhanden. Das erste Schreiben (S. 3-7) ist ein Brief Leibnizens an Nalebranche, der sich weder bei Cousin, noch bei Gerhardt findet, alles Übrige sind Briefe von demselben an den P. Lelong, der bekanntlich ein intimer Freund von Malebranche war. Guhrauer hatte also nicht ganz Unrecht mit der Vermutung, $\mathrm{da} B$ an dieser Stelle „mehr Briefe von Malebranche zu sein scheinen, als die von Cousin herausgegebenen", täuschte sich aber in der Annahme, daß sich hier anch die von Cousin herausgegebenen Schreiben befänden. Diesen selben siebzehnten Brief der Korrespondenz zwischen dem deutschen und dem französischen Philosophen fand der Verfasser dieses nun noch an einer anderen Stelle, nämlich in der von dem Abbé Blampignon (1862) herausgegebenen „Correspondance inédite de Malebranche ${ }^{*}$. Daß weder Cousin noch Gerhardt auch hiervon etwas wissen, erklärt sich wobl daraus, daß Blampignons Schrift den Haupttitel trägt: „Etude sur Malebranche“, dem dann allerdings die Bemerkung folgt: „d'après des documents inćdits suivie d'une correspondance inédite“. In einer „Etude sur Malebranche" vermutet man nicht sogleich, unedierte Briefe von Jeibniz zu finden. Woher nun diese „Correspondance inédite"? Blampignon teilt $\mathrm{S}$. IV des ${ }_{n}$ Avertissement" mit, daß er diese gebe "telle qu'on la trouve dans le second volume du travail d'Adry". 
Diese Adryscho „Arbeit" ist ein zweibändiges druckfertiges Manuskript, das sich ebenfalls in der „Bibliothèque nationale ${ }^{*}$ zu Paris befindet. Der vollständige Titel des zweiten Bandes lautet: „Vie de Nicolas Malebranche. T'ome II. I Contenant ses Lettres et celles de Plusieurs Savants, qui n'ont point été imprimées | A Paris 1791." Infolge der Stürme der Revolution ist das Werk Manuskript geblieben. Es scheint demnach, daß nach Adrys Tode einige seiner Manuskripte von Durand de Lançon gekauft worden sind, während Adry das erwähnte druckfertige Manuskript, um es vor dem Untergang zu retten, selbst der Bibliothek vermacht haben wird. -

Verfasser dieses erlaubt sich, den nicht uninteressanten Brief von Leibniz an Malebranche nochmals zum Abdruck zu bringen, da er weder in den bisherigen Leibniz-Ausgaben noch bei Cousin steht, die Blampignonsche Studie auch nur wenig verbreitet ist und es schließlich nicht unnötig erscheint, die Textvarianten anzugeben, die bei einer künftigen Leibniz-Ausgabe zu berücksichtigen sein dürften. Der Brief steht bei Durand de Lançon, wie erwähnt, auf S. 3-7, bei Blampignon auf S. $76-79$, bei Adry auf S: 143 bis 147. Auf die Angabe der blos orthographischen Abweichungen darf hier verzichtet werden; im allgemeinen sei nur bemerkt, daß Blampignon die bei Adry meist fehlenden Accente konsequent durchgeführt hat.

Lettre de Leibniz au P. Malebranche. Mon révérend Père,

Hanover, 1er Janvier 1700.

Je commence par le remercîment que je vous dois pour votre beau livre de l'Amour de Dieu. ${ }^{4}$ ) Il me semble que vous convenez avec les idées que j'en ai, et dont j'avois marqué quelque chose dans ma précédente. ${ }^{5}$ ) Si on donnoit des définitions, les disputes cesseroient bientôt. C'est pourquoi je tàchai d'en donner de la justice, de la sagesse, de la charité, et de la béatitude en parlant

4) Dieses Werk war nach der Angabe Andrés (La Vie du R. P. Malebranche. Paris 1886. S. 282) Ende 1697 oder Anfang 1698 im Drucke erschienen.

5) S. S. 357f. der Gerhardt'schen Ausgabe. 
Zur Gesch. d. Briefwechsels zwischen Leibniz und Malebranche. 319

du droit de la nature dans ma préface du Codex juris gentium diplomaticus. ${ }^{6}$ ) Je trouve ce même 'défaut dans la philosophie, et quelquefois $m \hat{e ̂ m e}^{7}$ ) dans les mathématiques. ${ }^{8}$ )

Ce que j'avois écrit à M. Bernouilli de Groningue, et qui l'avoit converti, sur l'estime des forces, n'a pas été imprimé. C'étoit ${ }^{9}$ ) des lettres que nous échangions, et qu'il aura communiqué à $M$. le marquis de l'Hôpital.

Ce n'est pas la première fois que j'ai réussi à persuader par lettres; mais cela n'est pas ordinaire, et encore moins de convaincre les gens par des livres, surtout lorsqu'ils ${ }^{10}$ ) ont pris parti publiquement; car peu de gens sont capables de cette sincérité sur ce chapitre que vous possédez avec tant d'autres belles qualités, et dont vous avez donné des preuves publiques. Les lettres pourtant paraissent plus propres à gagner ceux qui nous sont contraires que les livres, car elles intéressent moins ce point d'honneur; qui joue son rôle ${ }^{11}$ ) lor's même qu'on n'y pense point. Le tête à tête est le plus commode pour conférer sur la philosophie, mais des gens comme moi, qui se trouvent dans des endroits éloignés des grandes villes, ont le malheur de ne pouvoir profiter, par ce moyen, des pensées des excellents hommes dont Paris ou Londres abondent, et à qui on n'oserait ni ne doit demander qu'ils se donnent la peine de s'expliquer par lettres; ce qui surtout a lieu, mon révérend Père, à votre égard. Vous et autres personnes d'un mérite extraordinaire êtes chargés de l'instruction du genre humain, et vous employeriez mal votre temps, si vous vouliez vous appliquer à instruire des particuliers en écrivant des lettres $\left.{ }^{12}\right)$. Il n'en est pas de même de moi, car mes pensées n'étant pas encore assez fixées en système mis par ordre, je trouve du profit dans les objections et réflexions

9) S. Bd. IV der Dutensschen Ausgabe der Werke von Leibniz; ein Teil dieses „Vorworts" ist auch bei Gerhardt (III, 386-89) abgedruckt.

7) ,même" feblt bei Blampignon.

\&) Dieser Satz ist bei Bl. zum nächsten Absatz gezogen.

9 Bei Bl. „C'étoient".

10) Hier hat das Adry'sche Manuskript den Schreibfebler „lorsqu'il" wofür bei Lançon und Bl. das richtige „lorsqu'ils" stebt.

i) Lançon bat ,jeu" statt „róle".

12) Adry fāngt hier einen neuen Abschnitt an. 
que je rencontre dans les lettres de mes amis. Je prends plaisir de voir les différents biais dont on prend les mêmes choses; et cherchant à satisfaire ì un chacun (supposé qu'il cherche sincèrement la vérité), je trouve ordinairement des nouvelles ouvertures, lesquelles ne changean $t^{13}$ ) rien au fond de la chose, lui donnent toujours un plus grand jour. Ainsi je $n^{\prime} y^{14}$ ) ai jamais perdu mon temps.

Il passa ici, il y a près de trois mois ${ }^{15}$ ) une personne d'esprit et de savoir, qui alloit en Italie, en compagnie du fils de M. le comte de Guiscar. Il me marqua, ce me semble, qu'il avoit l'honneur d'être connu de vous. Je suis fort touché du malheur arrivé à cette belle compagnie; M. le marquis de Guiscar et M. d'Avennes étant morts à Vienne de la petite vérole. Toutes les fois que je pense à ces sortes d'accidents, je suis en colère contre les médecins (quoique d'ailleurs je les estime fort, mais de loin, tant que je puis), c'est qu'il semble qu'on devroit savoir le mojen de guérir les maladies assez ordinaires, qui ne consistent que dans les humeurs.

C'e que M. le marquis de l'Hôpital m'avoit envoyé sur le problème de $\mathrm{M}$. Fatio $\mathrm{le}^{16}$ ) Duillier, ayant été envoyé d'abord à. Leipzig, comme je lui marquai dans ma réponse, a été inséré depuis dans le journal qui s'y fait. Ce que je vous supplie de lui témoigner ${ }^{17}$ ), avec mes recommandations, et d'ajouter qu'on désapprouve fort, dans la société royale d'Angleterre, la manière dont Fatio en a usé à mon égard ${ }^{18}$ ). Nous avons appris de plus qu'au lieu d'avoir méprisé le problème de $M$. Bernouilli (comme il fait semblant), il l'a ${ }^{19}$ ) cherché inutilement, avec beaucoup d'application

13) Bl. hat den Druckfehler: „changent“.

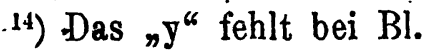

15) Bl. hat „ans"; die Lesart von Adry und Lançon ist wohl die richtige.

19): Liançon hat „de Duillier".

17) Malebranche, war mit l'Hôpital befreundet, der zunächst sein Schüler gewesen war; vgl. Fontenelle (Euvres Bd. V. Paris 1766) Eloge de M. le Marquis de l'Hộpital. S. 77.

18) Es handelt sich um den bekannten Prioritätsstreit zwvischen Leibniz und Newton, in dem Fatio eine wenig rühmliche Rolle spielte.

19) Das Manusk़ript Adrys hat den Schreibfehler "la cherche" [sc. le problème]. Dafür hat schon Lançon riçtig eingesetzt: ,l'a cherchéc. Bl. ist diese so naheliegende Verbesserung entgangen, er druckt: „la (sic) cherche“. 
Zur Gesch. d. Briefwechsels zwischen Leibniz und Malebranche. 321

fort longtemps. Ces manières peu sincères et peu convenables font déshonneur aux sciences. Je souhaite que dans votre nouvelle édition de la Recherche de la vérité, vous fassiez distinguer les additions ou changements, du reste, soit par la diversité des types ou par d'autres caractères, afin qu'on la puisse remarquer plus aisément, car il y aura sans doute des choses considérables. ${ }^{20}$ ) Je m'imagine que M. le marquis de l'Hôpital travaillera à son ouvrage nouveau, qui sera de conséquence, comme tout ce qu'il nous donne. ${ }^{21}$ ) Je suis avec zèle, mon révérend Père, votre très humble et très obéissant serviteur,

Leibniz.

20) Malebranche hat diesen Rat nur teilweise befolgt. Verfasser dieses möchte nicht verfehlen, an dieser Stelle darauf hinzuweisen, daß es auch heute noch an einer kritischen Ausgabe der „Recherche de la vérité", die zu des Verfassers Lebzeiten in sechs von einander abweichenden Ausgaben erschien, fehlt. Für das Genauere sei auf des Verfassers Schrift über Malebranches Erkenntnislehre verwiesen, die demnächst erscheinen wird.

21) Das „neue Werk" ist de l'Hôpitals "Traité analytique des Sections coniques et de la construction des lieux géométriques". l'H. starb 1704, ließ dieses Werk aber druckfertig zurück. Es erschien im Jahre 1707, vgl. Montucla. Histoire des Mathématiques Bd. II S. 398. 